Bull. Austral. Math. Soc.

$43 \mathrm{~A} 20,43 \mathrm{~A} 07,43 \mathrm{~A} 15$

VoL. 66 (2002) [69-73]

\title{
ORDER STRUCTURE ON CERTAIN CLASSES OF IDEALS IN GROUP ALGEBRAS AND AMENABILITY
}

\author{
YUJI TAKAHASHI
}

Let $G$ be a separable, locally compact group and let $\mathcal{J}_{d}(G)$ be the set of all closed left ideals in $L^{1}(G)$ which have the form $J_{\mu}=\left\{f-f * \mu: f \in L^{1}(G)\right\}^{-}$for some discrete probability measure $\mu$. It is shown that if $\mathcal{J}_{d}(G)$ has a unique maximal element with respect to the order structure by set inclusion, then $G$ is amenable. This answers a problem of G.A. Willis. We also examine cardinal numbers of the sets of maximal elements in $\mathcal{J}_{d}(G)$ for nonamenable groups.

Let $G$ be a locally compact group, and let $L^{1}(G)$ and $M(G)$ be the group and the measure algebras on $G$, respectively. As usual, we shall identify $L^{1}(G)$ with the ideal of $M(G)$ consisting of measures which are absolutely continuous with respect to left Haar measure. Let $P M(G)$ denote the set of probability measures in $M(G)$. For each $\mu \in P M(G)$, define

$$
J_{\mu}=\left\{f-f * \mu: f \in L^{1}(G)\right\}^{-}
$$

and

$$
\mathcal{J}(G)=\left\{J_{\mu}: \mu \in P M(G)\right\} .
$$

Then $J_{\mu}$ is a closed left ideal in $L^{1}(G)$ and $\mathcal{J}(G)$ is partially ordered by set inclusion. We also consider two classes of ordered subsets $\mathcal{J}_{a}(G)$ and $\mathcal{J}_{d}(G)$ of $\mathcal{J}(G)$ defined by

$$
\mathcal{J}_{a}(G)=\left\{J_{\mu}: \mu \in P M_{a}(G)\right\}
$$

and

$$
\mathcal{J}_{d}(G)=\left\{J_{\mu}: \mu \in P M_{d}(G)\right\}
$$

where $P M_{a}(G)$ and $P M_{d}(G)$ denote respectively the sets of absolutely continuous and discrete probability measures in $M(G)$.

Willis [9, Theorem 1.2 (b)] characterised the amenability of $G$ in terms of the order structure of $\mathcal{J}(G)$ or $\mathcal{J}_{a}(G)$ as follows: For a separable(that is, second countable), locally compact group $G, \mathcal{J}_{a}(G)$ (or $\mathcal{J}(G)$ ) has a unique maximal element if and only if $G$ is amenable. It was also shown that $\mathcal{J}_{d}(G)$ has a unique maximal element whenever $G$ is a

Received 3rd January, 2002

The author wishes to thank the referee for some helpful comments.

Copyright Clearance Centre, Inc. Serial-fee code: 0004-9727/02 \$A2.00+0.00. 
separable, amenable, locally compact group ([9, Proposition 1.3$])$. However the following problem remained open ([9, Problem 6.1]: If $G$ is a separable, locally compact group and if $\mathcal{J}_{d}(G)$ has a unique maximal element, then is $G$ amenable? In this note we resolve this problem affirmatively. It is also shown that $\mathcal{J}_{d}(G)$ has uncountably many maximal elements for some class of nonamenable groups.

Let $L_{0}^{1}(G)$ be the augmentation ideal of $L^{1}(G)$, that is

$$
L_{0}^{1}(G)=\left\{f \in L^{1}(G): \int_{G} f=0\right\} .
$$

It is well known that $L_{0}^{1}(G)$ coincides with the closed linear span of $\left\{f-f * \delta_{x}\right.$ : $\left.f \in L^{1}(G), x \in G\right\}$, where $\delta_{x}$ denotes the point mass at $x \in G$ ([6, Section 1, Proposition 1] or [7, Proposition 3.6.11]). In the following, this fact will be used repeatedly.

The following theorem shows that the answer to Willis' problem is affirmative. Our proof of the theorem also reproduces a result of Willis [9, Theorem $1.2(\mathrm{~b})]$.

THEOREM 1. Let $G$ be a separable, locally compact group and let $X$ be one of $\mathcal{J}(G), \mathcal{J}_{a}(G)$, and $\mathcal{J}_{d}(G)$. If $X$ has a unique maximal element, then $G$ is amenable.

Proof: Assume that $X$ has a unique maximal element, $J_{\mu}$ say. We claim that $f-f * \delta_{x} \in J_{\mu}$ for each $x \in G$ and $f \in L^{1}(G)$. This is valid when $X=\mathcal{J}(G)$ or $X=\mathcal{J}_{d}(G)$. In fact, since $f-f * \delta_{x} \in J_{\delta_{x}}$ and every element of $X$ is contained in a maximal one ([9, Theorem 1.2(a)]), we have $f-f * \delta_{x} \in J_{\delta_{x}} \subseteq J_{\mu}$. Now suppose that $X=\mathcal{J}_{a}(G)$, and let $\left\{u_{\lambda}\right\}_{\lambda \in \Lambda}$ be a bounded approximate identity for $L^{1}(G)$ such that $u_{\lambda} \in P M(G)$ for each $\lambda \in \Lambda$. (We may take a sequential bounded approximate identity because of the separability of $G$.) Since $f-f * u_{\lambda} * \delta_{x} \in J_{u_{\lambda} * \delta_{x}}$ and $u_{\lambda} * \delta_{x} \in L^{1}(G)$, we also have

$$
f-f * u_{\lambda} * \delta_{x} \in J_{u_{\lambda} * \delta_{x}} \subseteq J_{\mu}
$$

for each $\lambda \in \Lambda$. Hence it follows from the closedness of $J_{\mu}$ that

$$
f-f * \delta_{x}=\lim _{\lambda}\left(f-f * u_{\lambda} * \delta_{x}\right) \in J_{\mu}
$$

as desired. Since $L_{0}^{1}(G)$ is equal to the closed linear subspace generated by $\left\{f-f * \delta_{x}\right.$ : $\left.f \in L^{1}(G), x \in G\right\}$, our claim implies that $L_{0}^{1}(G) \subseteq J_{\mu}$. But the converse inclusion relation is clear, and so we have $L_{0}^{1}(G)=J_{\mu}$. Notice now that $J_{\mu}$ has a bounded right approximate identity. Indeed it is easy to verify that $\left\{u_{\lambda}-u_{\lambda} *\left(\sum_{i=1}^{n} \mu^{i}\right) / n\right\}_{(\lambda, n) \in \Lambda \times \mathbb{N}}$ is a bounded right approximate identity for the left ideal $J_{\mu}$ whenever $\left\{u_{\lambda}\right\}_{\lambda \in \Lambda}$ be a bounded approximate identity for $L^{1}(G)$. Thus $L_{0}^{1}(G)\left(=J_{\mu}\right.$ ) has a bounded right approximate identity. Therefore we may apply a result of Reiter [5] to conclude that $G$ is amenable. REMARKs 1. (1) Following [8] we say that $\mu \in P M(G)$ is ergodic by convolutions if $\lim _{n \rightarrow \infty}\left\|f *\left(\sum_{i=1}^{n} \mu^{i}\right) / n\right\|_{1}=0$ for all $f \in L_{0}^{1}(G)$. Rosenblatt [8, Proposition 1.9] showed 
that if there exists a probability measure on a locally compact group $G$ which is ergodic by convolutions, then $G$ is $\sigma$-compact and amenable. It is obvious that $\mu \in P M(G)$ is ergodic by convolutions if and only if $L_{0}^{1}(G)=J_{\mu}$. Recall also that if $G$ is a $\sigma$-compact locally compact group, then every element in $\mathcal{J}(G)$ (or $\mathcal{J}_{a}(G)$ ) is contained in a maximal one (see [9]). These facts may be combined with [8, Proposition 1.9 and Theorem 1.10] to show that the following conditions (i), (ii), and (iii) are equivalent whenever $G$ is a $\sigma$-compact locally compact group:

(i) $G$ is amenable;

(ii) there exists an absolutely continuous probability measure on $G$ which is ergodic by convolutions;

(iii) $\mathcal{J}(G)$ (or $\mathcal{J}_{a}(G)$ ) has a unique maximal element.

(2) The proof of Theorem 1 yields that if $G$ is a separable, locally compact group, then $\mathcal{J}_{d}(G)$ has a unique maximal element if and only if $L_{0}^{1}(G) \in \mathcal{J}_{d}(G)$. Thus it is still true that if $G$ is a separable, locally compact group, then the amenability of $G$ is equivalent to each of the following conditions (ii)' and (iii)':

(ii)' there exists a discrete probability measure on $G$ which is ergodic by convolutions;

(iii) $\mathcal{J}_{d}(G)$ has a unique maximal element.

The statement that $\mathcal{J}_{d}(G)$ has a unique maximal element does not necessarily imply the separability of $G$. Indeed, let $G$ be the Bohr compactification of $\boldsymbol{Z}$. It is then easily shown that $G$ is a compact Abelian group which is not separable. Since $G$ has a countable dense subset (for example, the image of $\boldsymbol{Z}$ under the canonical continuous homomorphism of $\boldsymbol{Z}$ to $G$ ), it follows from $\left[8\right.$, Corollary 1.14] that $L_{0}^{1}(G) \in \mathcal{J}_{d}(G)$. We can also obtain some examples of $\sigma$-compact locally compact groups for which $L_{0}^{1}(G) \notin \mathcal{J}_{d}(G)$.

The argument used in Theorem 1 may be applied to show that there exist many maximal elements in $\mathcal{J}(G), \mathcal{J}_{a}(G)$, and $\mathcal{J}_{d}(G)$ for some nonamenable groups. The following result gives a partial improvement of Theorem 1 .

TheOrem 2. Let $G$ be a separable, connected, locally compact group which is nonamenable. Then $\mathcal{J}_{d}(G)$ (or $\mathcal{J}(G)$ ) has uncountably many maximal elements.

Proof: We shall give the proof for $\mathcal{J}_{d}(G)$ only. The proof for $\mathcal{J}(G)$ is exactly the same. Suppose that the set of maximal elements in $\mathcal{J}_{d}(G)$ has at most countably many elements $\left\{J_{\mu_{n}}\right\}_{n \geqslant 1}$. We shall prove that $G$ is amenable. Now define

$$
H_{n}=\left\{x \in G: J_{\delta_{x}} \subseteq J_{\mu_{n}}\right\}
$$

for each $n$. Then $H_{n}$ is a closed subgroup of $G$. Indeed, $H_{n}$ is closed because the mapping $a \rightarrow f * \delta_{a}$ of $G$ into $L^{1}(G)$ is continuous for each $f \in L^{1}(G)$. That $H_{n}$ is a subgroup of $G$ follows immediately from the relations

$$
f-f * \delta_{x y}=f-f * \delta_{x}+\left\{\left(f * \delta_{x}\right)-\left(f * \delta_{x}\right) * \delta_{y}\right\}
$$


and

$$
f-f * \delta_{x^{-1}}=-\left\{\left(f * \delta_{x^{-1}}\right)-\left(f * \delta_{x^{-1}}\right) * \delta_{x}\right\}
$$

where $f \in L^{1}(G)$ and $x, y \in G$. Since every ideal in $\mathcal{J}_{d}(G)$ is contained in a maximal element $\left(\left[9\right.\right.$, Theorem 1.2(a)]), we also have $G=\bigcup_{n \geqslant 1} H_{n}$. Thus Baire category theorem implies that $H_{n_{0}}$ is open for some $n_{0}$. Noting now that $G$ is connected, we have

$$
G=H_{n_{0}}=\left\{x \in G: J_{\delta_{x}} \subseteq J_{\mu_{n_{0}}}\right\} \text {. }
$$

But then $L_{0}^{1}(G)=J_{\mu_{n_{0}}}$, and so $G$ is amenable, as desired.

We can also prove the following result on cardinal numbers of the set of maximal elements in $\mathcal{J}_{a}(G)$.

THEOREM 3. Let $G$ be a separable, connected, nonamenable locally compact group. Then $\mathcal{J}_{a}(G)$ has infinitely many maximal elements.

Proof: Assume that the set of maximal elements in $\mathcal{J}_{a}(G)$ consists of finitely many elements $\left\{J_{\mu_{i}}\right\}_{i=1}^{m}$. It will be shown that $G$ is amenable. Choose and fix $x \in G$. Let $\left\{u_{n}\right\}_{n \in N}$ be a sequential bounded approximate identity for $L^{1}(G)$ such that $u_{n} \in P M(G)$ for each $n \in N$. Since $u_{n} * \delta_{x} \in L^{1}(G)$ for every $n, J_{u_{n} * \delta_{x}} \subseteq J_{\mu_{i_{n}}}$ for some $i_{n}\left(1 \leqslant i_{n} \leqslant m\right)$ ([9, Theorem 1.2(a)]), and so we have

$$
N=\bigcup_{i=1}^{m}\left\{n \in N: J_{u_{n} * \delta_{x}} \subseteq J_{\mu_{i}}\right\}
$$

Hence there exist some $i \quad(1 \leqslant i \leqslant m)$ and a subsequence $\left\{u_{n_{k}}\right\}$ of $\left\{u_{n}\right\}$ such that $J_{u_{n_{k}} * \delta_{x}} \subseteq J_{\mu_{i}}$ for all $k$. Since $\lim _{k \rightarrow \infty}\left(f-f * u_{n_{k}} * \delta_{x}\right)=f-f * \delta_{x}$ for each $f \in L^{1}(G)$, it follows from the closedness of $J_{\mu_{i}}$ that $J_{\delta_{x}} \subseteq J_{\mu_{i}}$. Thus we conclude that

$$
G=\bigcup_{i=1}^{m}\left\{x \in G: J_{\delta_{x}} \subseteq J_{\mu_{i}}\right\} .
$$

Now the argument used in the proof of Theorem 2 may be applied to show that $L_{0}^{1}(G)$ $=J_{\mu_{i_{0}}}$ and hence $G$ is amenable.

REMARKS 2. (1) It is well known that $S L(n, \boldsymbol{R})(n \geqslant 2)$ is a connected Lie group which contains $\mathbf{F}_{2}$ (the free group on two generators) as a discrete subgroup ([3, Proposition 3.2] or [4, Corollary 14.6]). Thus $S L(n, \boldsymbol{R})$ is nonamenable, and so Theorem 2 implies that $\mathcal{J}(S L(n, \boldsymbol{R}))$ and $\mathcal{J}_{d}(S L(n, \boldsymbol{R}))$ have uncountably many maximal elements. It also follows from Theorem 3 that $\mathcal{J}_{a}(S L(n, R))$ has infinitely many maximal elements. More generally Theorem 2 and Theorem 3 may be applied to every noncompact, connected, semisimple Lie group with finite centre (see [7, Theorem 8.7.6]).

(2) Both Theorem 2 for $\mathcal{J}(G)$ and Theorem 3 are also valid for all connected, nonamenable locally compact groups. These may be shown by applying the theorem of Kakutani and Kodaira which asserts that if $G$ is $\sigma$-compact, then it has a compact normal subgroup $K$ such that $G / K$ is separable (see [2] or [1, Theorem 8.7]). 


\section{REFERENCES}

[1] E. Hewitt and K.A. Ross, Abstract harmonic analysis I, 2nd Edition (Springer-Verlag, Berlin, Heidelberg, New York, 1979).

[2] S. Kakutani and K. Kodaira, 'Über das Haarsche Mass in der lokal bikompakten Gruppe', Proc. Imp. Acad. Tokyo 20 (1944), 444450.

[3] A.L.T. Paterson, Amenability, Math. Surveys and Monographs 29 (Amer. Math. Soc., Providence, RI, 1988).

[4] J.-P. Pier, Amenable locally compact groups (John Wiley and Sons, New York, 1984).

[5] H. Reiter, 'Sur certains idéaux dans $L^{1}(G)$ ', C.R. Acad. Sci. Paris Sér. A - B 267 (1968), 882-885.

[6] H. Reiter, $L^{1}$-Algebras and Segal algebras, Lecture Notes in Mathematics 231 (Springer-Verlag, Berlin, Heidelberg, New York, 1971).

[7] H. Reiter and J.D. Stegeman, Classical harmonic analysis and locally compact groups, London Math. Soc. Monographs 22 (Clarendon Press, Oxford, 2000).

[8] J. Rosenblatt, 'Ergodic and mixing random walks on locally compact groups', Math. Ann. 257 (1981), 31-42.

[9] G.A. Willis, 'Probability measures on groups and some related ideals in group algebras', J. Functional Analysis 92 (1990), 202-263.

Department of Mathematics

Hokkaido University of Education

Hakodate 040-8567

Japan

e-mail: ytakahas@cc.hokkyodai,ac.jp 